\title{
May 2014 Pulmonary Case of the Month: Stress Relief
}

\author{
Robert W. Viggiano, MD \\ Department of Pulmonary Medicine \\ Mayo Clinic Arizona \\ Scottsdale, AZ
}

\section{History of Present IIIness}

A 62 year old man was referred for an abnormal CT scan of the chest. He was found to have an abnormality in the lung as an incidental finding on a CT scan of the abdomen done 6 months earlier for abdominal pain. A CT-guided needle biopsy was performed but revealed only scant tissue and no diagnosis was made.

The patient was asymptomatic without dyspnea, wheezing or cough. He had no fevers, chills, history of pneumonia or sinus disease. He denied any symptoms of gastroesophageal reflux disease (GERD), regurgitation, dysphagia or aspiration.

\section{PMH, FH, SH}

The patient had a small melanoma excised from his arm several months earlier. Family history was noncontributory. He smoked a pack per day for 7 years but quit over 30 years earlier. He does not drink.

\section{Medications}

- Vitamins

- Mineral oil laxative

\section{Physical Examination}

Physical examination was unremarkable.

\section{Radiography}

A CT scan of the chest was performed (Figure 1). 


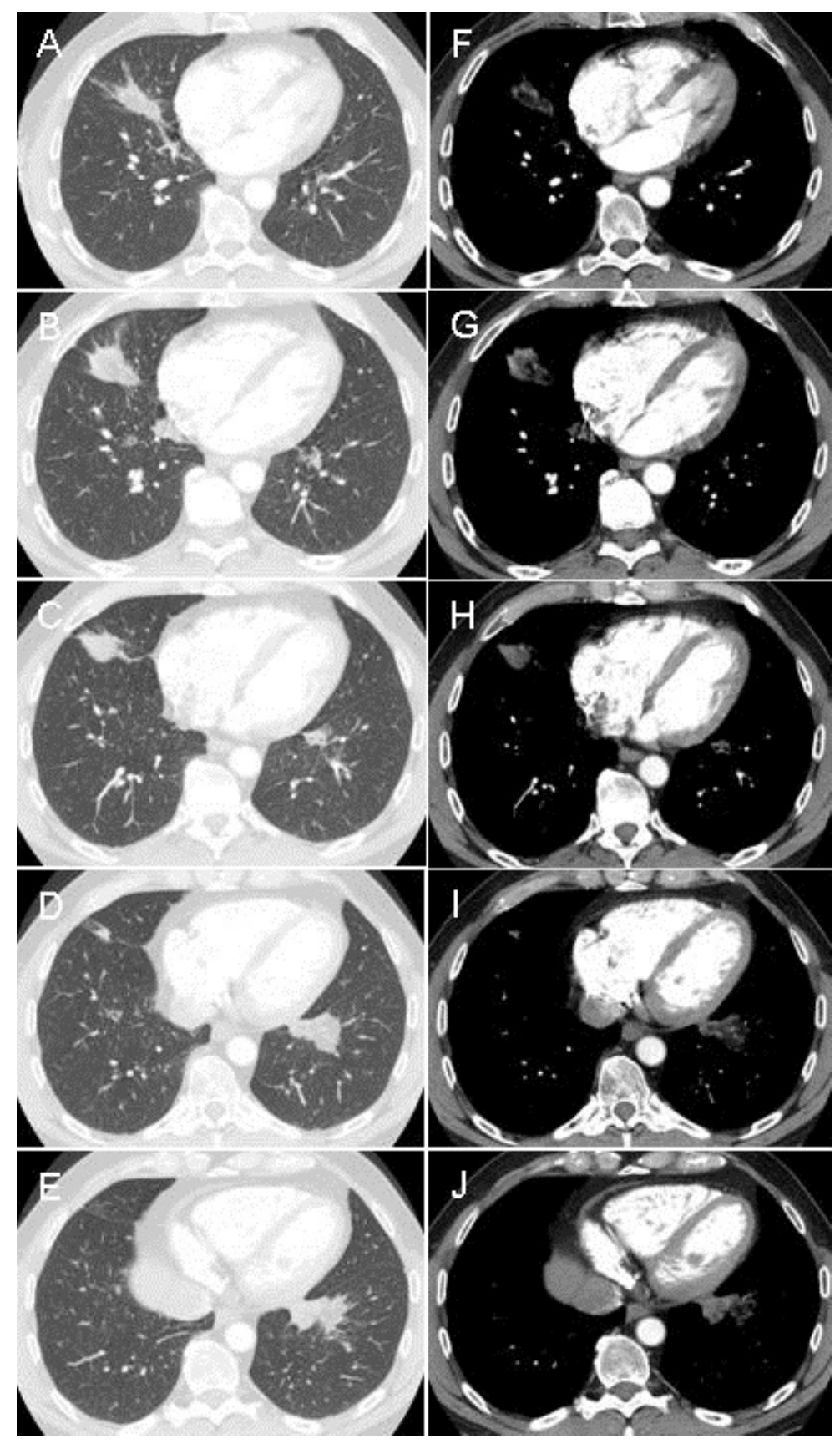

Figure 1. Representative images from the thoracic CT scan. Panels A-E: lung windows. Panels F-J: Corresponding soft tissue windows.

The thoracic CT shows which of the following abnormalities?

1. Left lower lobe mass

2. Mediastinal mass

3. Right middle lobe mass

4. 1 and 3

5. All of the above 


\section{Correct!}

\section{1 and 3}

There are two masses, one in the right middle lobe and the other in the left lower lobe. Both are relatively large at over $2 \mathrm{~cm}$. There is no significant mediastinal lymphadenopathy. The concern is that the lung lesions might be malignant.

Which of the following characteristics of lung masses are associated with malignancy?
1. Concave margins
2. Convex margins
3. Linear or angular shape
4. Lipid density
5. All of the above 


\section{Correct!}

\section{Convex margins}

The presence of a mass with a linear or angular shape or concave margins suggests the lesion is benign (1). In contrast, a spiculated or convex margin suggests malignancy.

A careful evaluation of the CT scan reveals that the lesions have a heterogeneous density (Figure 2).

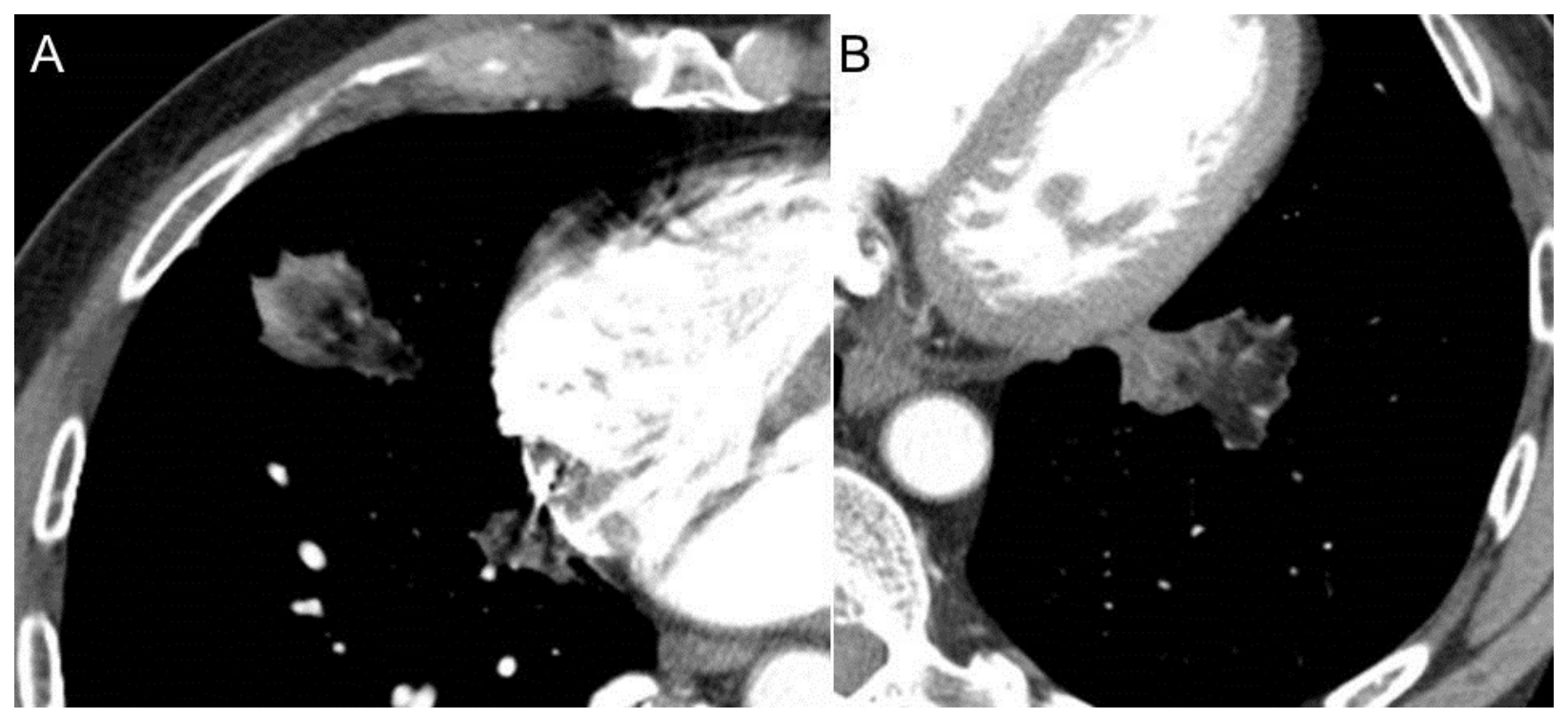

Figure 2. Close up of the right middle lobe lesion (Panel A) and the left lower lobe lesion (Panel B) showing heterogeneous densities.

Which of the following would be most consistent with a heterogeneous density?

1. Bacterial pneumonia

2. Lipoid pneumonia

3. Lung cancer

4. Metastatic melanoma

5. Pulmonary embolus 


\section{Correct! \\ 2. Lipoid pneumonia}

Of the choices listed only lipoid pneumonia has a density other than water. In this case the radiologist confirmed that the lesions showed low attenuation of $\sim-100$ Hounsfield units reflecting fat content.

Which of the following is true regarding lipoid pneumonia?

1. Aspiration of mineral oil tends to cause minimal lung inflammation

2. Most patients are elderly and asymptomatic

3. Radiologic manifestations can be variable

4. 1 and 3

5. All of the above 


\section{Correct! \\ 5. All of the above}

Exogenous lipoid pneumonia (ELP) results from repeated episodes of aspiration or inhalation of animal fat or mineral or vegetable oils over an extended period. ELP typically occurs in older debilitated patients with neurological or esophageal disease but can occur in patients without a predisposing anatomic or functional abnormality in swallowing. Patients are frequently asymptomatic with cough and dyspnea being the most common symptoms when present. ELP is uncommon with autopsy series reporting a frequency of only $1.0-2.5 \%(2)$.

Parenchymal abnormalities are dependent on the type, amount, frequency, and length of time of aspirated or inhaled oils or fats. Animal fats are hydrolyzed by lung lipases into free fatty acids that trigger a severe inflammatory reaction. The fatty acids either remain in the alveolar spaces or are phagocytosed by macrophages that then migrate to the interlobular septa leading to fibrosis. In contrast, mineral oil and vegetable-based oils tend to cause minimal to mild inflammatory reactions.

The radiologic manifestations are variable (table 1).

Table 1. Radiologic manifestations of lipoid pneumonia.

- Alveolar consolidations (57\%)

- Ground glass opacities (39\%)

-Alveolar nodules (23\%)

-Bilateral abnormalities (79\%)

-Predominant in the posterior and lower lobes (74\%)

- Hypodense $(71 \%)$

-Sparing of the subpleural zones (52\%)

The pathology of ELP is also variable but giant cell granulomas, alveolar and interstitial fibrosis, and chronic inflammation are often observed. Alveoli may be filled in by lipidladen macrophages with almost normal alveolar walls and septae. Advanced lesions show larger vacuoles and inflammatory infiltrates of the alveolar walls, bronchial walls, and septae.

Our patient was reassured and advised to discontinue the mineral oil. Several years later he was alive and doing well.

\section{References}

1. Li F, Sone S, Abe H, Macmahon H, Doi K. Malignant versus benign nodules at CT screening for lung cancer: comparison of thin-section CT findings. Radiology. 2004;233(3):793-8. [CrossRef] [PubMed]

2. Marchiori E, Zanetti G, Mano CM, Hochhegger B. Exogenous lipoid pneumonia. Clinical and radiological manifestations. Respir Med. 2011;105(5):659-66. [CrossRef] [PubMed] 